\title{
Short communication: Antimicrobial resistance and virulence genes of Enterococcus faecalis isolated from subclinical bovine mastitis cases in China
}

Feng Yang, Shidong Zhang, Xiaofei Shang, Xurong Wang, Zuoting Yan, Hongsheng Li, ${ }^{*}$ and Jianxi Li* Lanzhou Institute of Husbandry and Pharmaceutical Sciences of Chinese Academy of Agricultural Science, Lanzhou 730050, People's Republic of China

\section{ABSTRACT}

This study aimed to investigate the antimicrobial resistance and virulence genes of Enterococcus faecalis isolated from subclinical bovine mastitis cases in China. Enterococcus faecalis isolates were identified by $16 \mathrm{~S}$ rRNA amplification and sequencing. Antimicrobial susceptibility was determined by the disc diffusion method. Antimicrobial resistance and virulence genes were tested by PCR. Overall, E. faecalis was recovered from 81 of 1,787 (4.5\%) mastitic milk samples. The isolates showed high resistance against tetracycline $(87.7 \%)$ and erythromycin $(79.0 \%)$. The most prevalent resistance genes found in the E. faecalis were tetK $(96.3 \%)$, tet $L$ (79.0\%), and tetM $(87.7 \%)$ for tetracycline and ermC (97.5\%) for erythromycin. Moreover, gelE (70.4\%), esp (85.2\%), efaA (91.4\%) were the most common virulence genes. This is the first report to characterize E. faecalis recovered from subclinical bovine mastitis cases in China.

Key words: Enterococcus faecalis, bovine mastitis, antimicrobial resistance, virulence genes

\section{Short Communication}

Bovine mastitis is the most common and costly disease affecting dairy cattle throughout the world (Yang et al., 2016). Although mastitis control programs such as improved milking hygiene have led to considerable progress in controlling contagious mastitis pathogens, these procedures are less effective against environmental pathogens such as Enterococcus faecalis, which represents a major environmental mastitis-causing pathogen (Oliver and Pighetti, 2002; Elhadidy and Elsayyad, 2013).

Received February 11, 2018.

Accepted June 28, 2018.

*Corresponding authors: lihsheng@sina.com and lijianxigs@126 .com
Antimicrobials are commonly implemented for mastitis prevention and control. Unfortunately, the therapeutic result is limited due to the antimicrobial resistance of pathogens (Saini et al., 2012). Recently, phenotypic and genotypic resistance to several antimicrobials, including erythromycin (ermA, ermB, and erm C) and tetracycline (tetK, tet $L$, tet $M$, tet $O$, and tetS), have been widely documented in E. faecalis isolated from food-producing animals (Diarra et al., 2010; Šeputienè et al., 2012; Klibi et al., 2015). Moreover, several virulence factors have been described in $E$. faecalis, including gelatinase, hyaluronidase, aggregation substance, enterococcal surface protein, cytolysin, endocarditis-specific antigen, and collagen-binding cell wall protein (Martín-Platero et al., 2009; Perin et al., 2014). However, little is known about the E. faecalis isolates from bovine mastitis cases. The purpose of the current study was to investigate the antimicrobial resistance and virulence genes of E. faecalis isolated from subclinical bovine mastitis in China.

Mastitic milk samples of cows $(\mathrm{n}=1787)$ were collected from 62 commercial farms located in 15 provinces in China from April 2015 to November 2017 (Figure 1). Subclinical bovine mastitis was confirmed by the California mastitis test. After delivery to the laboratory, milk samples were inoculated onto $5 \%$ sheep blood agar plates and cultivated at $37^{\circ} \mathrm{C}$ for 24 to $48 \mathrm{~h}$. Presumptive colonies based on morphology and Gram strain were further identified by PCR and sequencing. Briefly, the bacterial genomic DNA was extracted using the Bacterial DNA Kit (Omega Bio-Tek, Norcross, GA) according to the manufacturer's recommendation (http: //omegabiotek.com/store/product/bacterial-dna-kit/). The 16S rRNA gene was amplified by PCR with the Bacterial 16S rDNA PCR Kit Fast (800; Takara, Shiga, Japan) in accordance with the manufacturer's instructions (http://catalog.takara-bio.co.jp/PDFS/rr182a_j .pdf). Subsequently, DNA sequence data for the first $500 \mathrm{bp}$ of the $16 \mathrm{~S}$ rRNA amplified gene were identified by the GenBank database comparison using the 


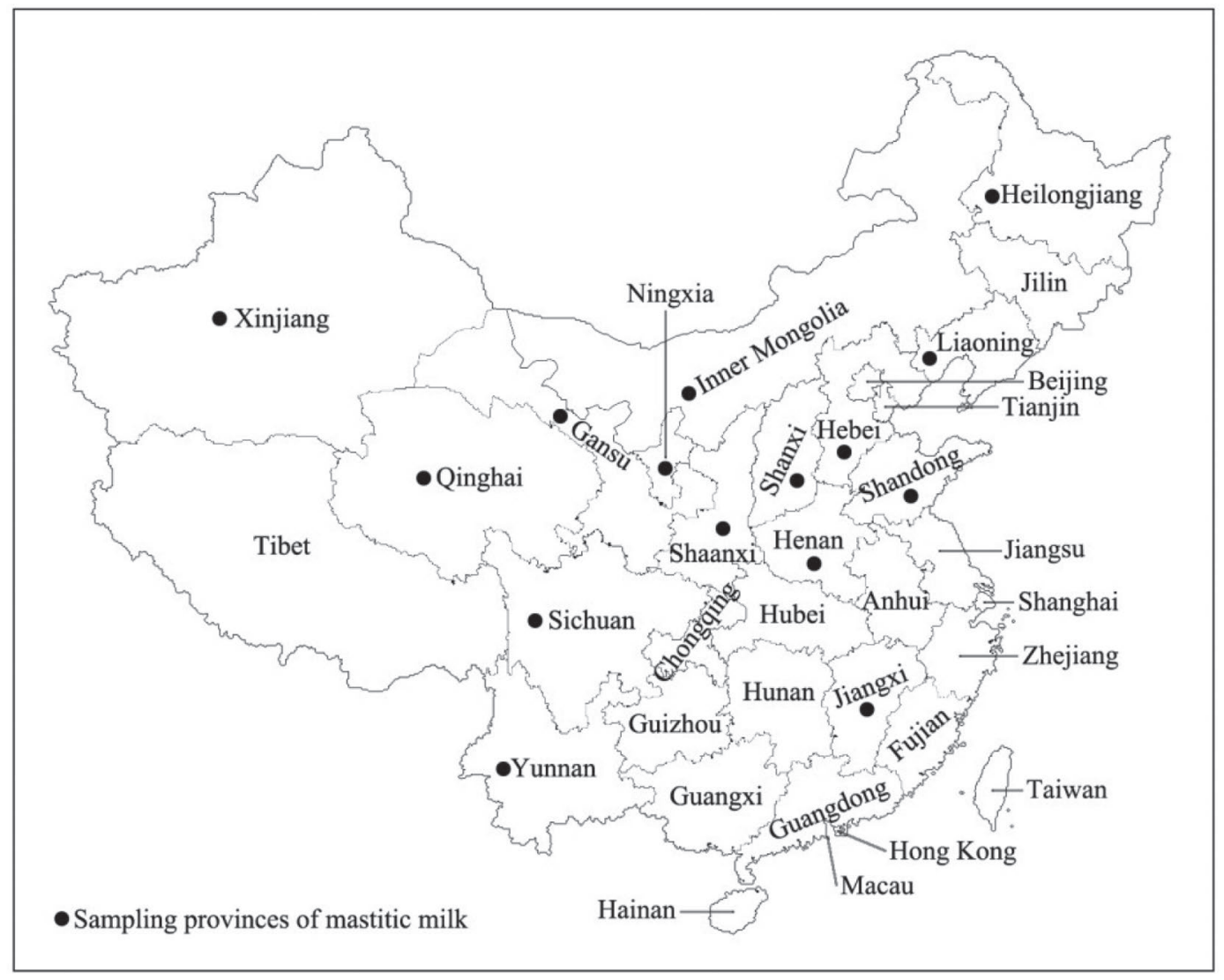

Figure 1. Sampling regions (provinces in China) involved in this study.

BLAST program (https://blast.ncbi.nlm.nih.gov/Blast .cgi). Sequences with 98 to $100 \%$ identity to sequences deposited in public domain databases were considered to be positive identification.

Antimicrobial susceptibility was performed by disc diffusion method on Mueller-Hinton agar (Oxoid, Basingstoke, Hampshire, United Kingdom) according to the recommendations of Clinical and Laboratory Standards Institute (CLSI, 2013). The panel of antimicrobial agents (Oxoid) included penicillin (10 U), vancomycin $(30 \mu \mathrm{g})$, erythromycin $(15 \mu \mathrm{g})$, tetracycline $(30 \mu \mathrm{g})$, ciprofloxacin $(5 \mu \mathrm{g})$, nitrofurantoin $(300 \mu \mathrm{g})$, rifampicin (5 $\mu \mathrm{g})$, chloramphenicol $(30 \mu \mathrm{g})$, quinupristin/dalfopristin $(15 \mu \mathrm{g})$, and linezolid $(30 \mu \mathrm{g})$. Staphylococcus aureus ATCC 25923 was used as quality control strain. Multidrug-resistant E. faecalis was defined as an isolate that was resistant to at least 3 antimicrobial agents belonging to different antimicrobial classes (Magiorakos et al., 2012).

Resistance genes of erythromycin (ermA, ermB, and erm $C$ ) and tetracycline (tetK, tetL, tet $M$, tet $O$, and tetS), as well as virulence genes gelE (gelatinase), hyl (hyaluronidase), asa1 (aggregation substance), esp (enterococcal surface protein), cylA (cytolysin), efaA (en- docarditis-specific antigen), and ace (collagen binding cell wall protein) were detected by PCR amplification with specific primers as described previously (Petersen and Dalsgaard, 2003; Amaral et al., 2017). Overall, 81 $(4.5 \%)$ E. faecalis isolates were detected from 1,787 mastitic milk samples. The antimicrobial susceptibility of the isolates against 10 antimicrobial agents is shown in Table 1. The E. faecalis isolates showed resistance against erythromycin $(79.0 \%)$, tetracycline $(87.7 \%)$, quinupristin/dalfopristin $(23.5 \%)$, rifampicin (18.5\%), chloramphenicol (14.8\%), and ciprofloxacin (11.1\%). Multidrug resistance was found in 15 (18.5\%) isolates. However, all the isolates were susceptible to vancomycin, nitrofurantoin, and linezolid. Moreover, 4 isolates were susceptible to all tested antimicrobial agents.

The most common antimicrobial resistance found in our study was to tetracycline and erythromycin. Thus, the resistance-associated genes of tetracycline (tetK, tetL, tet $M$, tet $O$, and tetS) and erythromycin (ermA, ermB, and erm C) were detected and results are summarized in Table 1 . Genes tetK, tet $L$, tet $M$, and tet $S$ were found in 78 (96.3\%), 64 (79.0\%), 71 (87.7\%), and $14(17.3 \%)$ of the E. faecalis isolates, respectively. Likewise, $21(25.9 \%)$ and $79(97.5 \%)$ of the isolates 


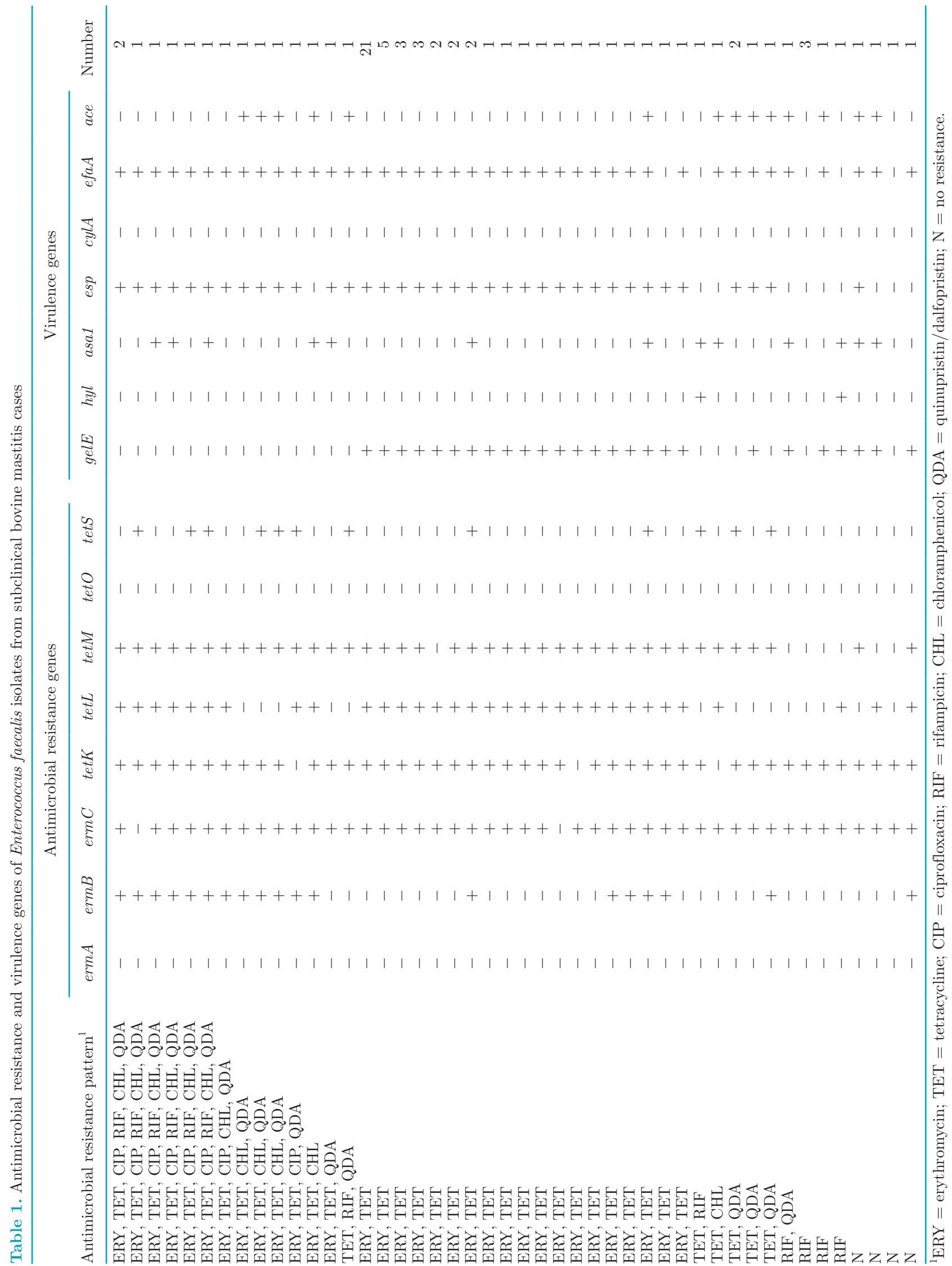


contained ermB and erm $C$, respectively. Notably, all 71 $(87.7 \%)$ tetracycline-resistant E. faecalis isolates carried resistance genes tetK $(97.2 \%)$, tetL $(85.9 \%)$, tetM $(97.2 \%)$, and tetS $(19.7 \%)$ alone or in combination. Moreover, out of the 64 (79.0\%) erythromycin-resistant isolates, $18(28.1 \%)$ and $62(96.9 \%)$ of the isolates harbored ermB and erm $C$ alone or in combination. However, erm $A$ and tet $O$ were not found in any of the isolates.

We also detected the virulence genes of the E. faecalis isolates (Table 1). The results showed that the gelE, hyl, asa, esp, efaA, and ace were detected in 57 (70.4\%), 2 (2.5\%), 20 (24.7\%), 69 (85.2\%), 74 (91.4\%), and $16(19.8 \%)$ of the isolates, respectively. However, cylA was not observed in any of the isolates. Moreover, $77(95.1 \%)$ of the isolates carried at least 2 virulence genes. Four isolates were negative for all tested virulence genes.

Enterococcus faecalis is an important environmental pathogen causing bovine mastitis. In this study, E. faecalis was detected in $4.5 \%$ of the mastitic milk samples. The occurrence was lower compared with previous studies, which reported the prevalence of E. faecalis in bovine mastitis cases to be $20.0 \%$ in Belgium (Devriese et al., 1999), 20.9\% in Czech Republic (Rysanek et al., 2009), and 19.5\% in Egypt (Elhadidy and Elsayyad, 2013 ), but higher than the $2.2 \%$ reported in Korea (Nam et al., 2010) and $0.2 \%$ in both Germany (Tenhagen et al., 2006) and the United States (Petersson-Wolfe et al., 2008). Variations in findings may be attributable to geographical discrepancies (Kasimoglu-Dogru et al., 2010).

Although Enterococcus spp. have low-level intrinsic resistance to aminoglycosides, lincosamides, and trimethoprim, emergence and prevalence of antimicrobial resistance is closely related to the use of antimicrobial agents (Klibi et al., 2015; Beukers et al., 2017). A variety of antimicrobials is available for therapeutic use in veterinary medicine in China (MOA, 2013). The high resistance against tetracycline and erythromycin found in our study could be due to long-term and widespread use of these antimicrobials on dairy farms. Previous studies have also reported high tetracycline and erythromycin resistance in E. faecalis isolated from food-producing animals (Diarra et al., 2010; Šeputienè et al., 2012).

Genetic determinants that may enhance the virulence of $E$. faecalis include antimicrobial resistance genes and a variety of virulence factors (Tejedor Junco et al., 2015). The antimicrobial resistance genes and virulence factors can be transferred to other bacteria, which pose a serious threat to public health through the food chain (Aslam et al., 2012; Iweriebor et al., 2015). In our study, the most common resistance genes found in
$E$. faecalis were tetK, tet $L$, and tet $M$ for tetracycline and $e r m C$ for erythromycin. Similarly, tetracycline genes were frequently found in tetracycline-resistant $E$. faecalis isolates from animals (Petersen and Dalsgaard, 2003; Diarra et al., 2010; Klibi et al., 2015). However, previous studies revealed that ermB was the most frequent gene found in enterococci isolated from human and animals (Petersen and Dalsgaard, 2003). The disparity could be due to the different origins of the erythromycin-resistant E. faecalis isolates. Notably, 4 isolates that were susceptible to tetracycline and erythromycin carried at least 1 of the tet and erm genes. This discrepancy can be attributed to the lack of expression of those resistance genes (Hammad et al., 2014). Moreover, in our study, gelE, esp, and efaA were the predominant virulence genes detected in the E. faecalis isolates. These findings agree with other recent studies that reported these virulence genes were frequently observed in E. faecalis isolates from different sources (Aslam et al., 2012; Iweriebor et al., 2015; Nateghian et al., 2016). The gelE gene is responsible for production of gelatinase, which is a metalloproteinase hydrolyzing casein, hemoglobulin, insulin, fibrinogen, collagen, gelatin, as well as various proteins or peptides. The esp and efa $A$ genes contribute to the colonization and persistence of enterococci in infections (ChajęckaWierzchowska et al., 2016). Furthermore, the presence of esp gene is reported to be highly associated with biofilm formation in enterococci (Diarra et al., 2010). The high prevalence of gelE, esp, and efaA in our study may indicate their potential virulence of $E$. faecalis in subclinical bovine mastitis in China.

In summary, although the occurrence of E. faecalis is relatively low in subclinical bovine mastitis cases in China, high-level phenotypic and genotypic resistance to tetracycline and erythromycin require government attention by way of comprehensive surveillance for the use of antimicrobial agents in dairy cattle. In addition, the frequent prevalence of gelE, esp, and $e f a A$ indicates that these virulence genes may play important roles in subclinical bovine mastitis pathogenesis in China.

\section{ACKNOWLEDGMENTS}

This work was supported by the National Key Research and Development Program of China (no. 2017YFD0502200, Lanzhou, People's Republic of China), the Dairy Industry Technology and Systems Projects (no. CARS-36, Lanzhou, People's Republic of China), and the Special Fund of Chinese Central Government for Basic Scientific Research Operations in Commonweal Research Institutes (No. 1610322017013, Lanzhou, People's Republic of China). 


\section{REFERENCES}

Amaral, D. M. F., L. F. Silva, S. N. Casarotti, L. C. S. Nascimento, and A. L. B. Penna. 2017. Enterococcus faecium and Enterococcus durans isolated from cheese: Survival in the presence of medications under simulated gastrointestinal conditions and adhesion properties. J. Dairy Sci. 100:933-949.

Aslam, M., M. S. Diarra, S. Checkley, V. Bohaychuk, and L. Masson. 2012. Characterization of antimicrobial resistance and virulence genes in Enterococcus spp. isolated from retail meats in Alberta, Canada. Int. J. Food Microbiol. 156:222-230.

Beukers, A. G., R. Zaheer, N. Goji, K. K. Amoako, A. V. Chaves, M. P. Ward, and T. A. McAllister. 2017. Comparative genomics of Enterococcus spp. isolated from bovine feces. BMC Microbiol. $17: 52$

Chajęcka-Wierzchowska, W., A. Zadernowska, and E. ŁaniewskaTrokenheim. 2016. Virulence factors, antimicrobial resistance and biofilm formation in Enterococcus spp. isolated from retail shrimps. Lebensm. Wiss. Technol. 69:117-122.

CLSI. 2013. Performance Standards for Antimicrobial Susceptibility Testing. CLSI document M100-S23. Clinical and Laboratory Standard Institute (CLSI), Wayne, PA.

Devriese, L. A., J. Hommez, H. Laevens, B. Pot, P. Vandamme, and F. Haesebrouck. 1999. Identification of aesculin-hydrolyzing streptococci, lactococci, aerococci and enterococci from subclinical intramammary infections in dairy cows. Vet. Microbiol. 70:87-94.

Diarra, M. S., H. Rempel, J. Champagne, L. Masson, J. Pritchard, and E. Topp. 2010. Distribution of antimicrobial resistance and virulence genes in Enterococcus spp. and characterization of isolates from broiler chickens. Appl. Environ. Microbiol. 76:8033-8043.

Elhadidy, M., and A. Elsayyad. 2013. Uncommitted role of enterococcal surface protein, Esp, and origin of isolates on biofilm production by Enterococcus faecalis isolated from bovine mastitis. J. Microbiol. Immunol. Infect. 46:80-84.

Hammad, A. M., T. Shimamoto, and T. Shimamoto. 2014. Genetic characterization of antibiotic resistance and virulence factors in Enterococcus spp. from Japanese retail ready-to-eat raw fish. Food Microbiol. 38:62-66.

Iweriebor, B. C., L. C. Obi, and A. I. Okoh. 2015. Virulence and antimicrobial resistance factors of Enterococcus spp. isolated from fecal samples from piggery farms in Eastern Cape, South Africa. BMC Microbiol. 15:136.

Kasimoglu-Dogru, A., Y. E. Gencay, and N. D. Ayaz. 2010. Prevalence and antibiotic resistance profiles of Enterococcus species in chicken at slaughter level; absence of $\operatorname{van} A$ and $\operatorname{vanB}$ genes in E. faecalis and E. faecium. Res. Vet. Sci. 89:153-158.

Klibi, N., R. Aouini, F. Borgo, L. B. Said, C. Ferrario, R. Dziri, A. Boudabous, C. Torres, and K. Ben Slama. 2015. Antibiotic resistance and virulence of faecal enterococci isolated from foodproducing animals in Tunisia. Ann. Microbiol. 65:695-702.

Magiorakos, A. P., A. Srinivasan, R. B. Carey, Y. Carmeli, M. E. Falagas, C. G. Giske, S. Harbarth, J. F. Hindler, G. Kahlmeter, and B. Olssonliljequist. 2012. Multidrug-resistant, extensively drugresistant and pandrug-resistant bacteria: An international expert proposal for interim standard definitions for acquired resistance. Clin. Microbiol. Infect. 18:268-281.
Martín-Platero, A. M., E. Valdivia, M. Maqueda, and M. MartínezBueno. 2009. Characterization and safety evaluation of enterococci isolated from Spanish goats' milk cheeses. Int. J. Food Microbiol. $132: 24-32$.

MOA. 2013. Ministry of Agriculture of the People's Republic of China. Announcement No.1997 of the Ministry of Agriculture. Accessed Mar. 17, 2018. http://www.moa.gov.cn/fwllm/zxbs/xzxk/spyj/ 201706/t20170606_5662448.htm (Online in Chinese).

Nam, H. M., S. K. Lim, J. S. Moon, H. M. Kang, J. M. Kim, K. C. Jang, J. M. Kim, M. I. Kang, Y. S. Joo, and S. C. Jung. 2010. Antimicrobial resistance of enterococci isolated from mastitic bovine milk samples in Korea. Zoonoses Public Health 57:e59-e64.

Nateghian, A., F. Fallah, Z. Daghighi, H. Goudarzi, A. Hashemi, and J. L. Robinson. 2016. Detection of virulence genes in resistant enterococci isolated from pediatric patients at high risk for nosocomial infections. Diagn. Microbiol. Infect. Dis. 85:260-262.

Oliver, S. P., and G. M. Pighetti. 2002. Mastitis pathogens: Environmental pathogens. Pages 1728-1734 in Encyclopedia of Dairy Sciences. University of Tennessee, Knoxville.

Perin, L. M. R. O. Miranda, S. D. Todorov, B. D. Franco, and L. A. Nero. 2014. Virulence, antibiotic resistance and biogenic amines of bacteriocinogenic lactococci and enterococci isolated from goat milk. Int. J. Food Microbiol. 185:121-126.

Petersen, A., and A. Dalsgaard. 2003. Species composition and antimicrobial resistance genes of Enterococcus spp, isolated from integrated and traditional fish farms in Thailand. Environ. Microbiol. 5:395-402.

Petersson-Wolfe, C. S., S. Adams, S. L. Wolf, and J. S. Hogan. 2008. Genomic typing of enterococci isolated from bovine mammary glands and environmental sources. J. Dairy Sci. 91:615-619.

Rysanek, D., M. Zouharova, and V. Babak. 2009. Monitoring major mastitis pathogens at the population level based on examination of bulk tank milk samples. J. Dairy Res. 76:117-123.

Saini, V., J. T. McClure, D. Léger, G. P. Keefe, D. T. Scholl, D. W. Morck, and H. W. Barkema. 2012. Antimicrobial resistance profiles of common mastitis pathogens on Canadian dairy farms. J. Dairy Sci. 95:4319-4332

Šeputienè, V., A. Bogdaitè, M. RužAuskas, and E. SužIedėLienè. 2012 Antibiotic resistance genes and virulence factors in Enterococcus faecium and Enterococcus faecalis from diseased farm animals: Pigs, cattle and poultry. Pol. J. Vet. Sci. 15:431-438.

Tejedor Junco, M. T., M. Gonzalez-Martin, N. F. Rodriguez Gonzalez, and C. Gutierrez. 2015. Identification, antimicrobial susceptibility, and virulence factors of Enterococcus spp. strains isolated from Camels in Canary Islands, Spain. Vet. Ital. 51:179-183.

Tenhagen, B. A., G. Köster, J. Wallmann, and W. Heuwieser. 2006. Prevalence of mastitis pathogens and their resistance against antimicrobial agents in dairy cows in Brandenburg, Germany. J. Dairy Sci. 89:2542-2551.

Yang, F., L. H. Liu, X. P. Li, J. Y. Luo, Z. Zhang, Z. T. Yan, S. D Zhang, and H. S. Li. 2016. Short communication: N-Acetylcysteine-mediated modulation of antibiotic susceptibility of bovine mastitis pathogens. J. Dairy Sci. 99:4300-4302. 